\title{
Disulfiram, a Ferroptosis Inducer, Triggers Lysosomal Membrane Permeabilization by Up-Regulating ROS in Glioblastoma
}

This article was published in the following Dove Press journal: OncoTargets and Therapy

\author{
Chen Qiu ${ }^{1-3, *}$ \\ Xin Zhang ${ }^{3,4, *}$ \\ Bin Huang ${ }^{3,4}$ \\ Shuai Wang ${ }^{3,4}$ \\ Wenjing Zhou ${ }^{3,4}$ \\ Chao $\mathrm{Li}^{3,4}$ \\ Xingang $\mathrm{Li}^{3,4}$ \\ Jian Wang ${ }^{3-5}$ \\ Ning Yang (D) $3,4,6$
}

'Department of Radiation Oncology, Qilu Hospital of Shandong University, Jinan, People's Republic of China; ${ }^{2}$ School of Medicine, Cheeloo College of Medicine, Shandong University, Jinan, People's Republic of China; ${ }^{3}$ Department of Neurosurgery, Qilu Hospital of Shandong University and Institute of Brain and Brain-Inspired Science, Shandong University, Jinan, People's Republic of China; ${ }^{4}$ Shandong Key Laboratory of Brain Function Remodeling, Jinan, People's Republic of China; ${ }^{5} \mathrm{~K}$. G. Jebsen Brain Tumor Research Center,

Department of Biomedicine, University of Bergen, Bergen, Norway; ${ }^{6}$ Department of Epidemiology and Health Statistics, School of Public Health, Shandong University, Jinan, People's Republic of China

*These authors contributed equally to this work
Introduction: Disulfiram (DSF), a drug used in the treatment of alcoholism since 1948, has been shown to have antitumor properties in various tumor types possibly due to the induction of a type cell death, ferroptosis, and the sensitization of cells to chemo- and radiotherapy. In this study, we explored the antitumor properties of DSF in glioblastoma (GBM) and investigated the underlying molecular mechanisms.

Methods: GBM cell lines U251 and LN229 were treated with DSF to assess cytotoxicity and activity of the molecule in vitro. Response of cells to treatment was examined using cell viability, flow cytometry, LDH release assay, immunofluorescence and Western blot analysis. Results: DSF inhibited cell growth of GBM U251 and LN229 cell lines in vitro in a concentration-dependent manner. Flow cytometry demonstrated that DSF caused G0-G1 growth arrest. DSF treatment led to increased ROS and lipid peroxidation levels relative to controls indicating the involvement of ferroptosis. Furthermore, DSF triggered lysosomal membrane permeabilization (LMP), a critical mechanism promoting cell death, in a ROSdependent manner. Finally, DSF enhanced radiosensitivity of U251 and LN229 cells.

Discussion: Our findings indicated that DSF induced ferroptosis and LMP and enhanced the radiosensitivity of GBM cells. Therefore, DSF might have efficient antitumor activity in the treatment of human GBM.

Keywords: disulfiram, ferroptosis, lysosomal membrane permeabilization, ROS, glioblastoma

\section{Introduction}

Glioblastoma (GBM) is the most malignant primary tumor of the brain. Despite an aggressive treatment regimen which includes surgery, chemo- and radiotherapy, the prognosis of GBM patients is still poor. Thus, new treatments are desperately sought after. ${ }^{1}$

Resistance to apoptosis is one of the most important factors leading to the treatment failure of GBM. ${ }^{2}$ Apoptosis, one of the most studied programmed cell death (PCD) pathways, is the target of many antitumor treatments. Moreover, other types of PCD, including autophagy, necroptosis and pyroptosis have been discovered and facilitated the search to overcome cancer treatment resistance. ${ }^{3}$ Ferroptosis is a newly discovered form of PCD. ${ }^{4}$ Characteristics include an accumulation of reactive oxygen species (ROS) resulting from intracellular iron accumulation and subsequent lipid peroxidation. Key morphological features of ferroptosis are shrunken mitochondria and increased density of the mitochondrial membrane. ${ }^{5}$
Correspondence: Ning Yang Email yangning@sdu.edu.cn 
The cystine/glutamate antiporter $\left(\mathrm{x}_{\mathrm{c}}{ }^{-}\right)$/glutathione (GSH)/glutathione peroxidase 4 (GPX4) axis is a critical pathway involved in regulating ferroptosis. ${ }^{6} \mathrm{xCT}$ (SLC7A11) light chain, a subunit of system $\mathrm{x}_{\mathrm{c}}{ }^{-}$, and GPX4, a selenoenzyme, are considered as key anti-ferroptosis proteins. ${ }^{7,8}$ Considering the role of ferroptosis in regulating cell death, ferroptosis might play an important role in tumorigenesis and tumor development. Erastin, a well-studied inducer of ferroptosis, has been shown to have remarkable anticancer activity in various tumor types. ${ }^{9-11}$ In addition, ferroptosis also enhances the antitumor properties of traditional chemo- and radiotherapy. ${ }^{9,12,13}$ As a result, ferroptosis is a promising target in anticancer treatment.

Cell death can also be induced by lysosomes, and this cell death type is called lysosomal membrane permeabilization (LMP). ${ }^{14}$ In LMP, impaired lysosomal membranes allow the release of specific lysosomal enzymes into the cytosol, resulting in the hydrolyzation of various organelles. ${ }^{15}$ Among the many factors triggering LMP, ROS is the most important. Considering that the level of ROS is up-regulated in ferroptosis, LMP might have a role in the process. A recent study demonstrated that ferroptosis is a lysosomal cell death process, ${ }^{16}$ which supports this possibility.

Disulfiram (DSF) is traditionally used for the treatment of alcoholism and has been used clinically for many years. ${ }^{17}$ In recent years, an increasing number of studies have shown that DSF has promising antitumor properties and enhances sensitivity to radio- and chemotherapy in breast, bladder, and colorectal cancers. ${ }^{18-20}$ However, the mechanisms underlying its antitumor activity are not fully understood. Here, we investigated the antitumor properties of DSF in GBM cell lines. Our study demonstrates that DSF inhibits cell growth through the induction of ROSdependent ferroptosis and LMP. Inhibiting ferroptosis counteracts LMP induced by DSF. These results highlight DSF as a promising antitumor agent for GBM and possibly other tumor types.

\section{Materials and Methods}

\section{Cell Culture}

GBM cell lines U251 and LN229 were purchased from the Culture Collection of the Chinese Academy of Sciences (Shanghai, China) and cultured in DMEM (ThermoFisher Scientific; Waltham, MA, USA) supplemented with 10\% fetal bovine serum (FBS; ThermoFisher Scientific).

\section{Cell Viability Assay}

U251 and LN229 cells $\left(1.0 \times 10^{4}\right.$ cells/well $)$ were plated into 96-well plates and incubated overnight. DSF $(0,5,10$, $15,20,30,40$, and $50 \mu \mathrm{M})$ was added to wells and cells were incubated with DSF for another $24 \mathrm{~h}$. Cell viability was assessed with the CCK-8 assay. CCK-8 solution was added to each well, and colorimetric analysis was performed on a microplate reader at $450 \mathrm{~nm}$ (PerkinElmer; San Jose, CA, USA).

\section{EdU Assay}

Cell proliferation was assessed with the EdU Assay Kit (Ribobio; Guangzhou, China) according to the manufacturer's instructions. Briefly, U251 and LN229 cells $(2.5 \times$ $10^{4}$ cells/well) were seeded into 24-well, flat-bottomed plates and treated with $5 \mu \mathrm{M}$ DSF or DMSO (vehicle control). Cells were incubated with EdU. Images were acquired under fluorescence microscopy (Leica DMi8; Wetzlar, Germany).

\section{Cell Cycle Analysis}

After treatment with $5 \mu \mathrm{M}$ DSF or DMSO, U251 and LN229 cells were collected through trypsinization and fixed with $70 \%$ ethanol overnight. Cells were rinsed with PBS and incubated with propidium iodide (PI) supplemented with RNase (Becton Dickinson, San Diego, CA). Cell cycle analysis was performed on a $\mathrm{C} 6$ flow cytometer (BD Biosciences; San Jose, CA, USA).

\section{Lipid Peroxidation and ROS Assay}

Intracellular levels of lipid peroxidation/ROS were assessed using BODIPY581/591 C11/CellRox green dye (ThermoFisher Scientific). BODIPY581/591 C11 or CellRox green dye ( $5 \mu \mathrm{M}$; diluted in DMEM with $10 \%$ fetal bovine serum) was added to U251 and LN229 cells pretreated with $5 \mu \mathrm{M}$ DSF or DMSO. Fluorescence was observed under confocal microscopy (Leica SP5 Confocal Microscope, Leica).

\section{Iron Assay}

The level of ferrous iron was tested by a colorimetric assay kit from Biovision (Milpitas, California, USA). Briefly, after treatment, U251 and LN229 cells were incubated with iron assay buffer. Supernatants were collected and incubated with iron probe. Then, samples were tested in a microplate reader at a wavelength of $593 \mathrm{~nm}$ (PerkinElmer). 


\section{Western Blot Analysis}

U251 and LN229 cells were treated with $2.5 \mu \mathrm{M}, 5 \mu \mathrm{M}$ and 10 $\mu \mathrm{M}$ DSF or DMSO for $24 \mathrm{~h}$. Cells were lysed in RIPA buffer (ThermoFisher Scientific) and lysates $(20 \mu \mathrm{g})$ were analyzed on SDS-PAGE. Western blot analysis was performed as previously described. ${ }^{21}$ The following antibodies were used: GPX-4, x-CT, GAPDH and $\gamma$-H2AX (Abcam; Cambridge, UK).

\section{Transmission Electron Microscopy}

Transmission electron microscopy was used to examine the ultrastructure of U251 and LN229 cells treated with $5 \mu \mathrm{M}$ DSF or DMSO. Experiments were carried out as described previously. ${ }^{21}$ Images were obtained using a JEM-1200EX II electron microscope (JEOL; Tokyo, Japan).

\section{Lysosomal Membrane Stability}

Lysosomal membrane stability was assessed through two assays, acridine orange (AO; Sigma-Aldrich, USA) staining and GFP-fused galectin 3 (Obio, Shanghai, China) transient transfection.

For AO staining, U251 and LN229 cells were incubated with $\mathrm{AO}(5 \mu \mathrm{g} / \mathrm{mL})$ for $15 \mathrm{~min}$, and treated with 5 $\mu \mathrm{M}$ DSF or DMSO for $60 \mathrm{~min}$. Fluorescence was detected under fluorescence microscopy with a Leica DMi8.

Transient transfections for GFP-fused galectin 3 were carried out with lipofectamine 2000 reagent (ThermoFisher Scientific). Transfected U251 and LN229 cells were treated with $5 \mu \mathrm{M}$ DSF or DMSO. Green dots in each treatment were counted under confocal microscopy with a Leica SP5 Confocal Microscope.

\section{LysoTracker Staining}

LysoTracker Red (66 nM; diluted in DMEM with $10 \%$ fetal bovine serum, ThermoFisher Scientific) was added to U251 and LN229 cells pretreated with $5 \mu \mathrm{M}$ DSF or DMSO. Nuclei were stained with DAPI (ThermoFisher Scientific). Fluorescence was observed under confocal microscopy with a Leica SP5 Confocal Microscope.

\section{Live/Dead Cell Viability Assay}

Cytotoxic effects were assessed using a Live/Dead cell viability assay kit (Thermo Fisher Scientific). U251 and LN229 cells were divided into four treatment groups: DMSO, $5 \mu \mathrm{M}$ DSF, 4 Gy irradiation at a dose rate of 1.8 $\mathrm{Gy} / \mathrm{min}$ in a linear accelerator (Primus $\mathrm{Hi}$, Siemens Medical Instruments; Berlin, Germany), and $5 \mu \mathrm{M}$ DSF combined with 4 Gy irradiation. Cells were incubated with a combination of $5 \mu \mathrm{L}$ calcein $\mathrm{AM}$ and $20 \mu \mathrm{L}$ ethidium homodimer-1 staining solution (diluted in DMEM with $10 \%$ fetal bovine serum). Green and red fluorescence were observed under fluorescence microscopy with a Leica DMi8 fluorescence microscope.

\section{Immunofluorescence}

DNA damage was determined using immunofluorescence staining of $\gamma-\mathrm{H} 2 \mathrm{AX}$ foci. U251 and LN229 cells were treated as described in 2.8. Cells were incubated with $\gamma$-H2AX antibody (Cell Signaling Technology; Danvers, MA, USA) and Alexa Fluor 594 conjugated goat anti-rabbit secondary antibody (Abcam; Cambridge, UK). Red dots corresponding to $\gamma$ H2AX were counted under a Leica TCS SP5 Confocal Laser Scanning Microscope (Leica Microsystem).

\section{Statistical Analysis}

Unpaired t-tests were performed using GraphPad Prism software program (Version 6.07; La Jolla, CA, USA). Results are presented as the mean \pm SE. $P$-values $<0.05$ were considered statistically significant.

\section{Results}

\section{DSF Inhibits Cell Growth of GBM Cells}

To begin to determine whether DSF might inhibit GBM, U251 and LN229 cells were treated with DSF in culture, and cell viability and proliferation assays were performed. Growth curves were generated for the two cell lines using colorimetric data from the CCK-8 assay and showed that cell viability of U251 and LN229 cells decreased under treatment with DSF in a dose-dependent manner (Figure 1A). The IC50 for U251 and LN229 was $30 \mu \mathrm{M}$ and $13.5 \mu \mathrm{M}$, respectively (Figure 1A). Thus, LN229 cells were more sensitive to DSF than U251 cells. EdU incorporation also showed that DSF inhibited cell growth. Treatment with $5 \mu \mathrm{M}$ DSF led to a decrease in the number of EdU positive cells compared with cells treated with DMSO $(38.67 \% \pm 2.028 \%$ vs $23.67 \% \pm 1.202 \%$ for U 251 ; $39.67 \% \pm 1.333 \%$ vs $13.00 \% \pm 1.155 \%$ for LN229; Figure $1 \mathrm{~B}$ and $\mathrm{C}$ ).

Flow cytometry was used to analyze cell cycle parameters of U251 and LN229 cells treated with DSF or the vehicle control DMSO. The percentage of cells in G0-G1 increased in cells treated with $5 \mu \mathrm{M}$ DSF relative to controls $(57.73 \%$ vs $63.05 \%$ for U251; $59.7 \%$ vs $66 \%$ for LN229); Figure 1D and E. All together, these results indicated that DSF inhibited growth of GBM cells in vitro. 
A

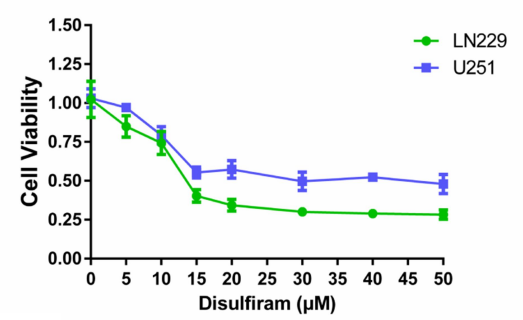

C

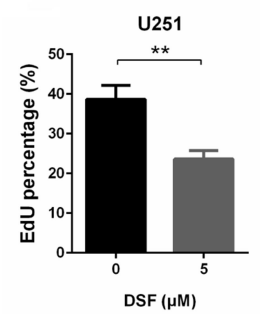

D

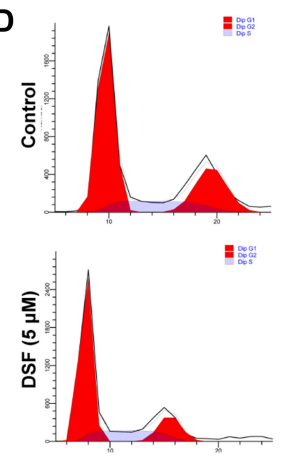

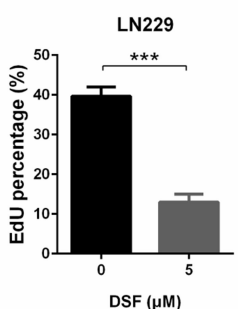

DSF ( $\mu$ M)
B

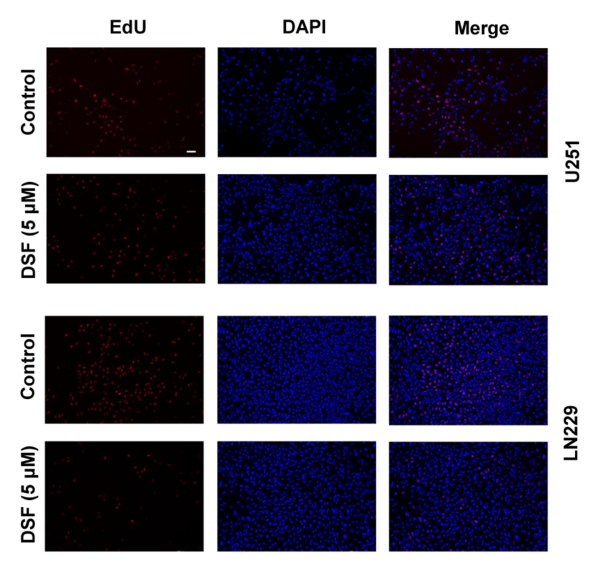

E
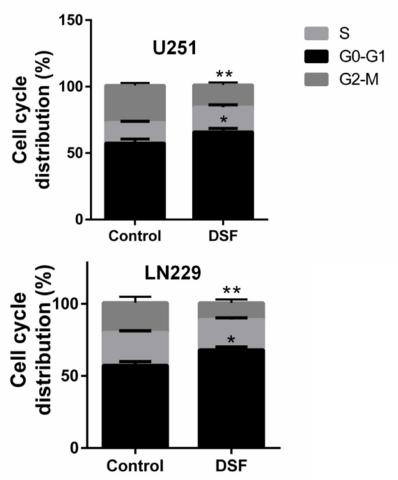

Figure I DSF inhibits cell growth of GBM cells (A) Growth curves generated with colorimetric data (O.D. $450 \mathrm{~nm})$ from the CCK-8 assay. U25I and LN229 cells were treated with increasing concentrations of DSF for $24 \mathrm{~h}$. The IC 50 for U25I and LN229 was $30 \mu \mathrm{M}$ and I3.5 $\mu \mathrm{M}$, respectively. (B) Fluorescence images for EdU incorporation at $5 \mu$ M DSF and DMSO (vehicle control) for $24 \mathrm{~h}$ in U25I and LN229 cells. (C) Quantification of EdU positive cells treated with DMSO (vehicle control) and $5 \mu$ M DSF in B. (D) Cell cycle analysis of U25I and LN229 cells treated with DMSO (vehicle control) and $5 \mu$ M DSF for $24 \mathrm{~h}$. (E) Quantification of cell cycle parameters G0-GI, S, and G2-M obtained from flow cytometric analysis in $D$. $* P<0.05 ; * P<0.01$; $* * * P<0.001$; scale bars $=50 \mu \mathrm{m}$.

\section{DSF Induces Ferroptosis in U25I and LN229 Cells}

We next investigated whether DSF induced ferroptosis in GBM cells. Up-regulation of lipid peroxidation is one of the characteristics of ferroptosis. Oxidation of the free radical sensor BODIPY ${ }^{581 / 591} \mathrm{C} 11$ dye shifts fluorescence of the dye from red to green. Under DSF treatment, red fluorescence of the dye decreased while green fluorescence increased in both U251 and LN229 cells (Figure 2A). These data suggested that DSF increased lipid peroxidation. CellRox Green Reagent, which detects oxidative stress, exhibited bright green fluorescence in DSF treated GBM cells. These results indicated that DSF led to increased total ROS in U251 and LN229 cells (Figure 2B and C). We also tested the levels of ferrous iron with a colorimetric assay kit and results showed that DSF increased levels of ferrous iron (Figure 2D). Levels of proteins that block ferroptosis, $\mathrm{xCT}$ and GPX-4, decreased in the presence of DSF in a dose-dependent manner (Figure 2E). Transmission electron microscopy also revealed the shrunken morphology of mitochondria in DSF treated GBM cells (red arrows, Figure 2F) and the enhanced density of mitochondrial membranes (Figure 2F). Iron chelator deferoxamine mesylate (DFO) and ferroptosis inhibitor ferrostatin-1 (Fer-1) were used to verify whether these results were iron- and ferroptosisdependent. We found that DFO and Fer-1 inhibited DSFinduced up-regulation of lipid peroxidation (Figure $2 \mathrm{G}$ ) and cytotoxic effect (Figure 2H). All together, these data demonstrated that DSF triggered ferroptosis in GBM.

\section{DSF Causes Lysosomal Membrane Permeabilization in GBM Cells}

A recent study demonstrated that ferroptosis is a lysosome-dependent cell death process. Lysosomal membrane permeabilization (LMP) is one mechanism inducing cell 
A
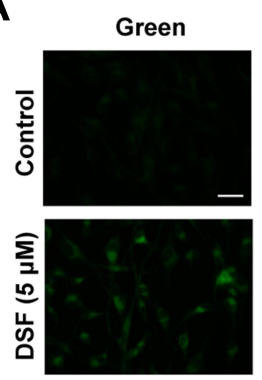

B

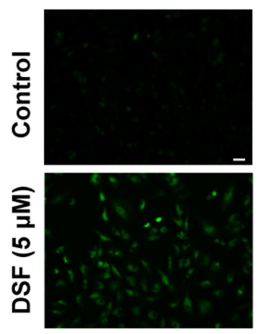

U251

E

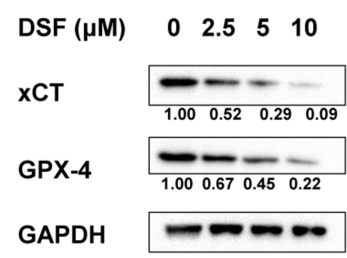

U251
Red
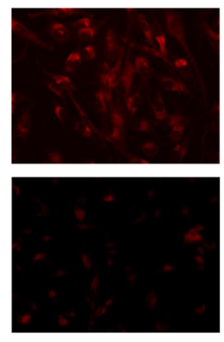

U251

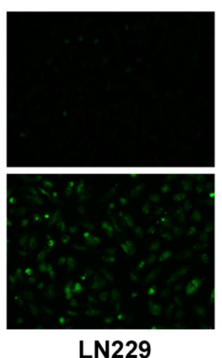

LN229

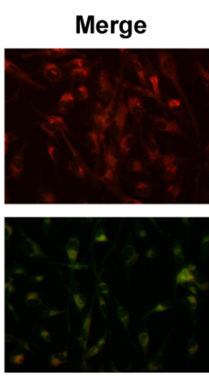

C

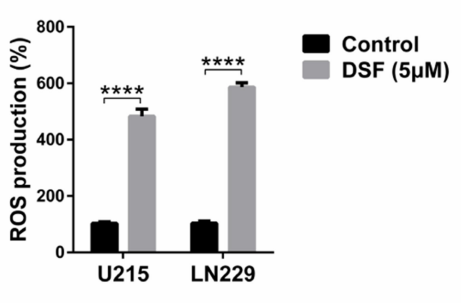

Red

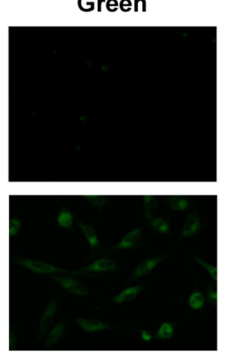

F

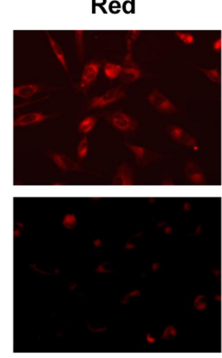

LN229

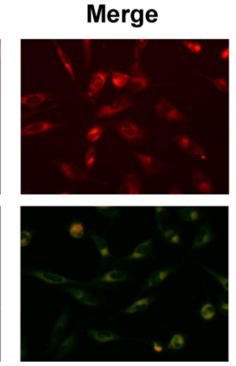

D

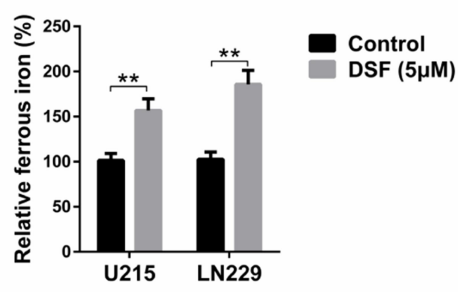

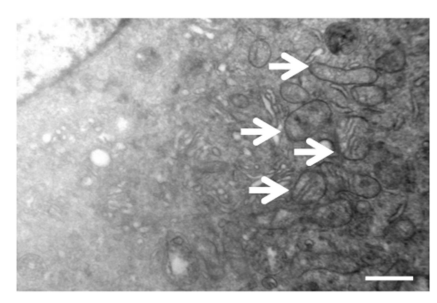

Control

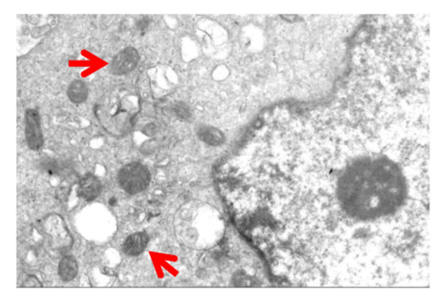

DSF $(5 \mu \mathrm{M})$

G

Control

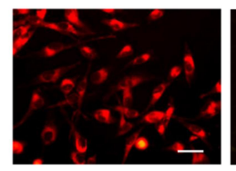

$\begin{array}{llll}0 & 2.5 & 5 & 10\end{array}$

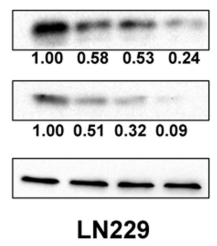

DFO+DSF

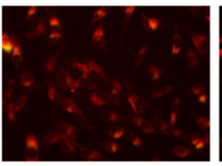

Fer-1+DSF

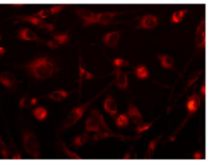

H

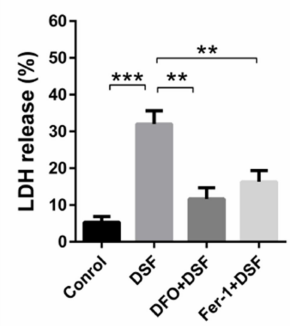

Figure 2 DSF induces ferroptosis in GBM cells. (A) Representative fluorescence images of BODIPY581/59I CII staining of U25I and LN229 cells treated with DMSO (vehicle control) and $5 \mu \mathrm{M}$ DSF for $24 \mathrm{~h}$. (B) Representative fluorescence images of CellRox Green staining of U25I and LN229 cells treated with DMSO (vehicle control) and $5 \mu$ M DSF. (C) Quantification of fluorescence in (B). (D) Levels of ferrous iron in U25I and LN229 cells treated with DMSO (vehicle control) and $5 \mu$ M DSF. (E) Western blot analysis of lysates prepared from U25I and LN229 cells treated with 0, 2.5, 5 and $10 \mu \mathrm{MDSF}$ for $24 \mathrm{~h}$ to detect levels of protein inhibitors of ferroptosis, $\mathrm{xCT}$ and GPX-4. GAPDH was used as a loading control. The xCT/GAPDH and GPX-4/GAPDH ratios were calculated using Imagej (National Institutes of Health, version I.52r). (F) Transmission electron microscopy image showing the ultrastructure of U25I cells treated with DMSO (vehicle control) or $5 \mu$ MDSF. Normal mitochondria in control cells highlighted with the white arrows; shrunken mitochondria and the enhanced density of mitochondrial membrane highlighted with red arrows in DSF treated U25I cells. (G) BODIPY ${ }^{581 / 591} \mathrm{CI}$ I staining of U25 I and LN229 cells treated with DMSO, 5 $\mu \mathrm{MDSF}, 50 \mu \mathrm{M}$ iron chelator deferoxamine mesylate (DFO) $+5 \mu \mathrm{MDSF}, 2 \mu \mathrm{M}$ Ferrostatin-I (Fer-I) $+5 \mu \mathrm{MDSF}$ (H) LDH release assay was used to test the cytotoxic effect of U25I and LN229 cells treated with DMSO, I $0 \mu$ MDSF, $50 \mu$ M iron chelator deferoxamine mesylate (DFO) $+10 \mu M D S F, 2 \mu M$ Ferrostatin-I (Fer-I) + I0 $\mu$ M DSF. **P<0.0 I; *** $2<0.00$ I; ****P< 0.000 I; Size bars in $(\mathbf{A}, \mathbf{B}$ and $\mathbf{G})=50 \mu \mathrm{m}$, in $(\mathbf{E})=0.5 \mu \mathrm{m}$.

death and the most studied form of lysosome-dependent cell death. When LMP occurs, lysosomal contents, including many acid proteases, leak into the cytoplasm, destroying critical proteins and resulting in cell death. We therefore tested whether DSF triggered LMP in GBM cells. Acridine orange (AO), a cell-permeant dye, is lysosomotropic, and emits red fluorescence at high concentrations and green fluorescence at low concentrations. Intact 
lysosomes emit red fluorescence after AO staining. When membranes are compromised, the concentration of $\mathrm{AO}$ decreased, and thus red fluorescence decreases. The red fluorescence of AO decreased in U251 and LN229 cells treated with DSF compared with untreated controls (Figure 3A).

Galectin 3, a $\beta$-galactoside binding lectin, accumulates on luminal glycoproteins when the membranes of endosomes or lysosomes are impaired. GBM cells were therefore transfected with a GFP-fused galectin 3 (EGFP-Gal3) plasmid to determine whether DSF induced LMP. EGFPGal3 appeared in fluorescent puncta in the DSF treated cells but only as a diffuse fluorescence in control cells (Figure 3B and C). LysoTracker Red is another lysosomotropic dye used to test whether lysosomes retain their function. Intact lysosomes have an acidic lumen which emit red fluorescence when stained with LysoTracker Red. When lysosomes are impaired, the intensity of the red fluorescence decreases. Red fluorescence decreased significantly in DSF-treated U251 and LN229 cells, indicating that DSF interfered with the function of lysosomes in GBM cells (Figure 3D). These data indicated that DSF led to LMP in GBM cells in culture.

\section{DSF Induces LMP in a ROS-Dependent Manner}

Many factors induce LMP, such as ROS and lysosomotropic reagents. Because DSF induced ROS in GBM cells, we next investigated whether DSF induces LMP in a ROS-
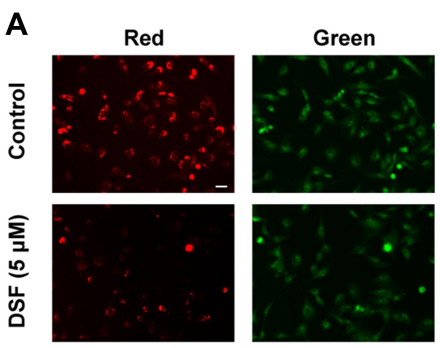

U251
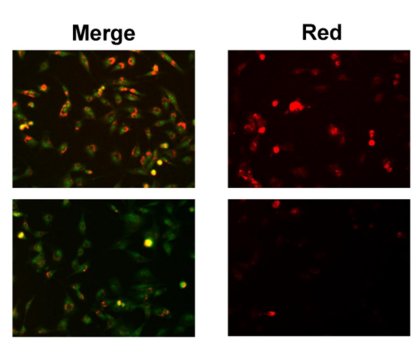

LN229

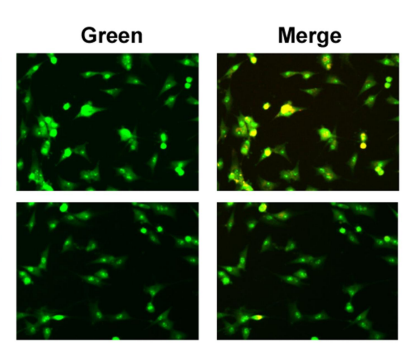

B

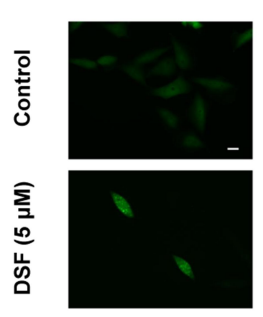

U251

D
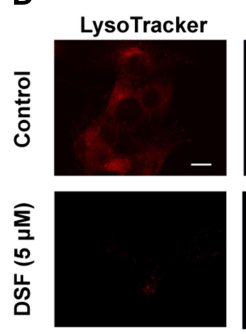
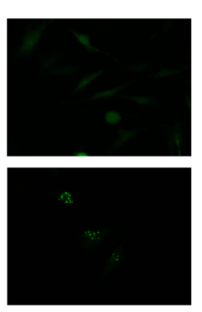

LN229
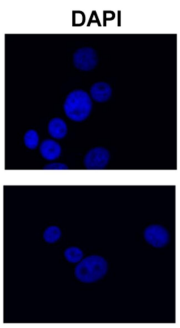

U251
C
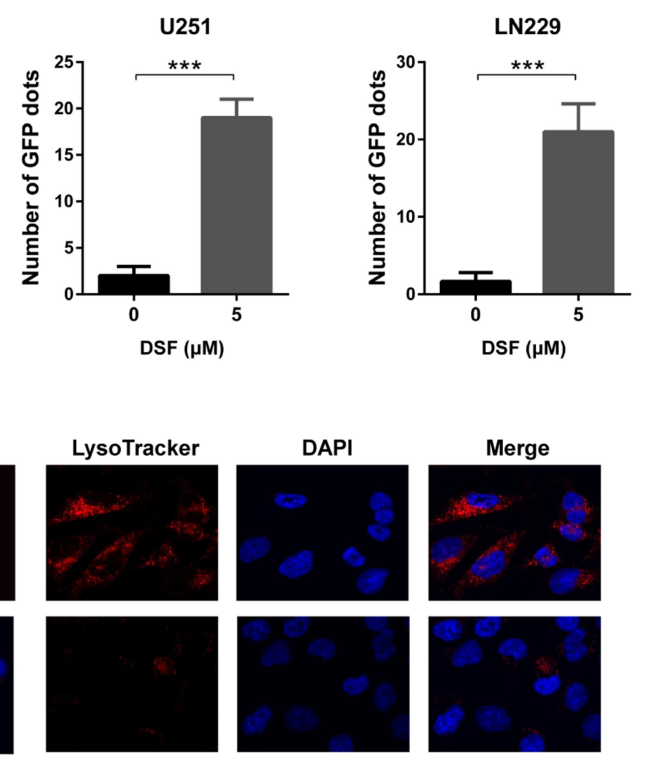

LN229

Figure 3 DSF induces lysosomal membrane permeabilization in GBM cells. (A) Fluorescence images of acridine orange staining of DSF treated U25I and LN229 cells compared with controls (DMSO, vehicle control). Red fluorescence is associated with high concentrations of acridine orange, while green fluorescence is associated with reduced concentrations of acridine orange. (B) Fluorescence images of U25I and LN229 cells treated with DSF and transfected with EGFP-Gal3 construct (green fluorescence). Green puncta result from the collection of EGFP-Gal3 collection on compromised lysosomes. (C) Quantification of green puncta in (B). (D) Fluorescence images of LysoTracker Red staining of DSF treated U25I and LN229 cells. $* * * P<0.001$; scale bars in $(\mathbf{A})=50 \mu \mathrm{m}$, in $(\mathbf{B}$ and $\mathbf{D})=10 \mu \mathrm{m}$. 
dependent manner. Cells were therefore exposed to glutathione (GSH), a ROS scavenger, to inhibit ROS production in GBM cells under treatment with DSF. In U251 and LN229 cells transfected with EGFP-Gal3, GSH prevented the appearance of green puncta induced by DSF treatment (Figure 4A and B). Cells were also treated with ferrostatin (Fer-1), a small molecule inhibitor of ferroptosis, to explore whether DSF-induced LMP was ferroptosis dependent. Fer-1 also prevented the increase in EGFP-Gal3 puncta induced by DSF in U251 and LN229 cells (Figure 4A and B). All together, these results indicated that DSF-induced LMP in GBM cells was ferroptosis dependent.

\section{DSF Increased Radiosensitivity in GBM Cells}

In our previous work, we found that Lys05, an LMP inducer, increased the radiosensitivity of U251 and
LN229 cells. In many other studies, DSF was also found to enhance the antitumor effect of radiotherapy. We therefore investigated whether DSF enhanced the radiosensitivity of GBM cells. Using a Live/Dead cell viability assay, we found that radiation combined with DSF led to increased cell death compared with radiation or DSF treatment alone (Figure 5A and $\mathrm{B}$ ).

DNA damage is the main cause of cell death in response to radiation, and double strand breaks (DSBs) are the most lethal of the various types of DNA damage induced by radiation exposure. We therefore used immunofluorescence staining of $\gamma$-H2AX, a well-known marker of DSBs, to determine whether DSF sensitized cells to radiation treatment. U251 and LN229 cells exposed to both radiation and DSF exhibited increased staining of $\gamma$ H2AX relative to cells treated with either DSF treatment alone or controls (Figure 5C and D). Western blot analysis of $\gamma-\mathrm{H} 2 \mathrm{AX}$ also showed that DSF combined with radiation

A
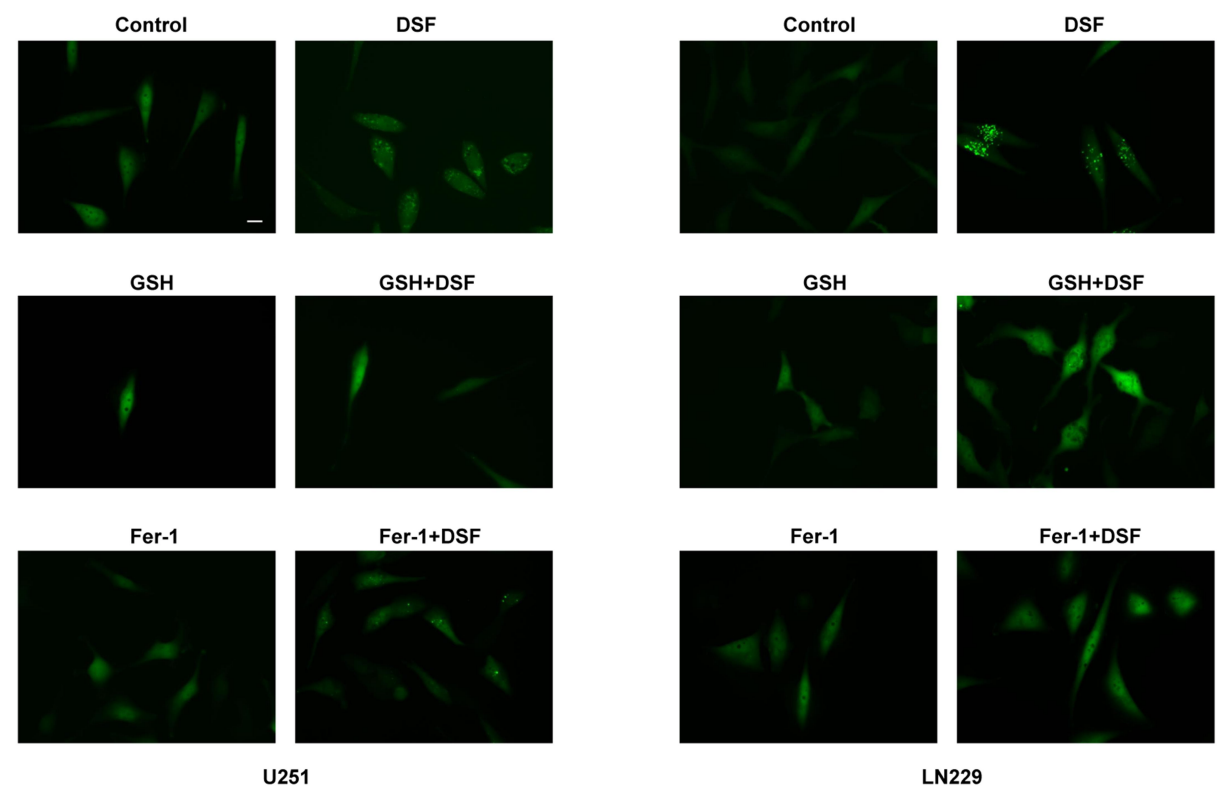

B
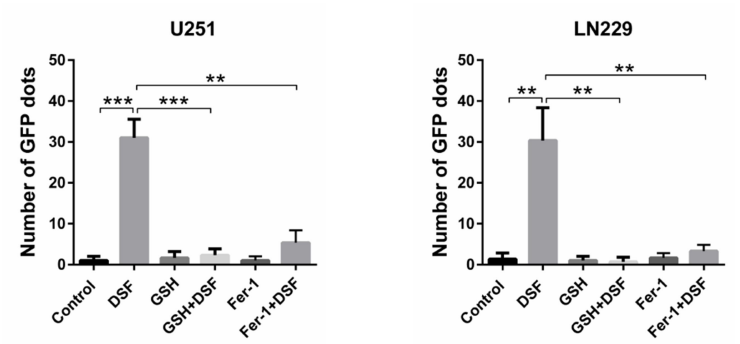

Figure 4 DSF induces LMP in a ROS-dependent manner. (A) Fluorescence images of cells transfected with EGFP-Gal3. U25I and LN229 cells were treated with DSF and inhibitors of ferroptosis, $5 \mathrm{mM} \mathrm{GSH}$ or $2 \mu \mathrm{M}$ ferrostatin-I (Fer-I), and compared with DSF treatment alone. (B) Quantification of green punta in $(\mathbf{A})$. $* * P<0.0 \mathrm{I}$; $* * * P<$ $0.00 \mathrm{I}$; size bars $=50 \mu \mathrm{m}$. 

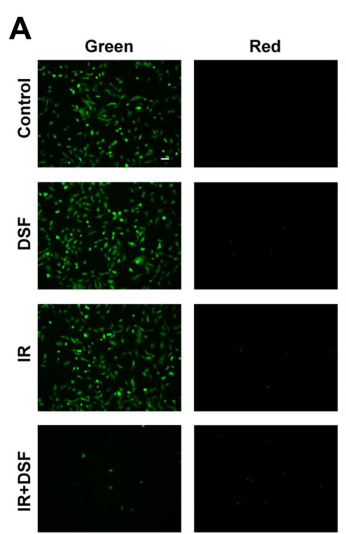

U251

C
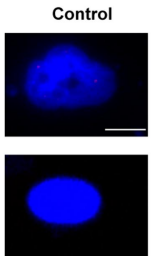

DSF
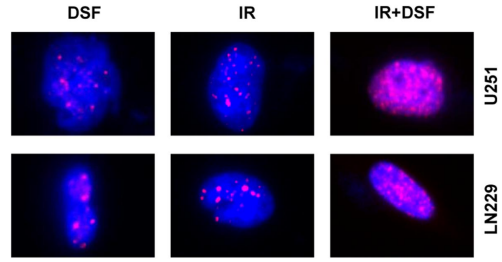

B
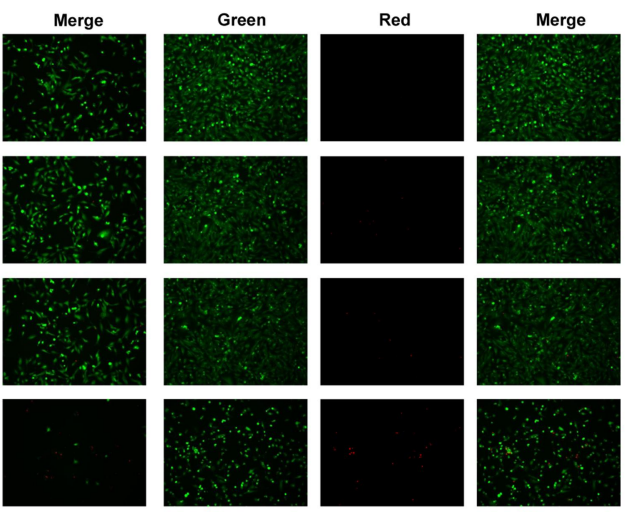

LN229

D
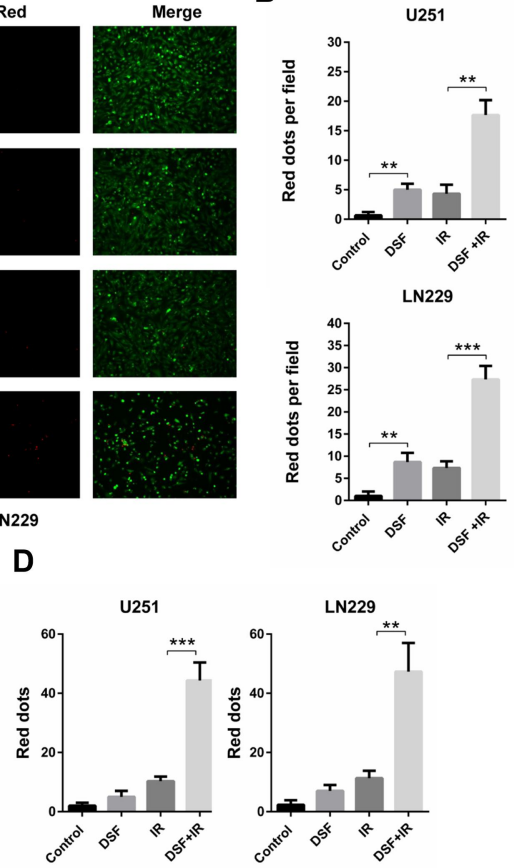

E

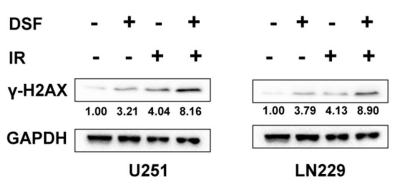

Figure 5 DSF enhances the radiosensitivity of GBM cells. (A) Fluorescence images of the Live/Dead staining assay (green fluorescence, live; red fluorescence, dead) performed on U25I and LN229 cells treated with DSF (5 $\mu$ M), radiation (4 GY, IR) or a combination of DSF and radiation. (B) Quantification of dead cells (red fluorescence) in (A). (C) Immunofluorescence staining of $\gamma-\mathrm{H} 2 \mathrm{AX}$ (red fluorescence) to determine the double strand breaks (DSBs) caused by DSF, radiation (IR) or a combination of DSF and radiation. (D) Quantification of red puncta associated with $\gamma-\mathrm{H} 2 \mathrm{AX}$ in (C). (E) Western blot analysis of lysates prepared from U25I and LN229 cells treated with DSF (5 $\mu \mathrm{M})$, radiation (4 GY, IR) or a combination of DSF and radiation. GAPDH was used as a loading control. The $\gamma$-H2AX/GAPDH ratio was calculated using ImageJ. **P < $0.0 \mathrm{I}$; $* * * \mathrm{P}<0.001$; size bars in $(\mathbf{A})=50 \mu \mathrm{m}$, in $(\mathbf{D})=10 \mu \mathrm{m}$.

led to more DNA damage compared with other groups (Figure 5E). All together, these data suggested that DSF enhanced radiosensitivity in GBM cells in culture.

\section{Discussion}

DSF has been used for the treatment of alcoholism since 1948.

An increasing number of studies have reported that DSF exhibits dramatic anticancer activity in various cancer cell types. DSF has also been shown to enhance the antitumor properties of chemotherapy and radiotherapy. ${ }^{18-20,22,23}$ Here, we showed that DSF displays efficient anti-glioma activity by decreasing cell viability, inhibiting cell proliferation and causing in G0-G1 cell cycle arrest. DSF also enhanced the radiosensitivity of GBM cells in vitro. Many studies have demonstrated that DSF possesses copper-dependent anticancer activity and that copper greatly enhances the antitumor efficacy of DSF. ${ }^{24}$ In fact, the $\mathrm{IC}_{50}$ of DSF in several tumor types was decreased when combined with copper. ${ }^{25}$ Further study is therefore warranted to determine whether the combination of DSF with copper is more efficient in triggering ferroptosis and LMP in GBM.

Resistance to apoptosis is one of the most important factors contributing to the failure of anti-cancer treatment.${ }^{26}$ Discovering new methods to overcome apoptotic resistance might tremendously advance the treatment of cancer. Ferroptosis is a newly-discovered mechanism of programmed cell death. ${ }^{3}$ Iron accumulation up-regulates ROS production, which results in lipid peroxidation and subsequent cell death. ${ }^{27}$ Most studies have shown that DSF kills cancer cells by causing apoptosis. ${ }^{20,28}$ Until now, only a single study has shown that DSF induces ferroptosis in cancer, specifically nasopharyngeal cancer. ${ }^{17}$ In our study, DSF treatment significantly increased the levels of lipid peroxidation and ROS in GBM cells. The protein levels of xCT and GPX-4, two important antioxidant proteins, were also decreased. Finally, the 
morphology of the mitochondria in the presence of DSF was consistent with the cellular process of ferroptosis. Thus, DSF could be classified as an inducer of ferroptosis in GBM cells.

ROS is also a well-known inducer of LMP. ${ }^{29}$ When LMP occurs, lysosomal contents, including many acid proteases, leak into the cytoplasm, resulting in cell death. ${ }^{30}$ Our work demonstrated that DSF also triggered LMP in U251 and LN229 cells and that GSH, a ROS scavenger, interfered with DSF-induced LMP. In addition, ferrostatin1, a small molecule inhibitor of ferroptosis, prevented DSF-induced LMP. Thus, ROS might be a key link between ferroptosis and LMP.

Despite aggressive treatment of GBM with a combination of surgery, and radio- and chemotherapy, patient prognosis remains poor. New therapies are urgently needed. Yet conventional drug development is time consuming and expensive. As a result, drug repurposing has gained increasing attention as an approach to the identification of viable anticancer therapies. ${ }^{31}$ In a previous study, we found that trifluoperazine, an antipsychotic drug in the treatment of schizophrenia, increased the radiosensitivity of GBM both in vitro and in vivo. ${ }^{21}$ Many drugs that have been used in the clinic for many years may have mechanisms of action other than their main indications, and these drugs have well-established clinical safety profiles. ${ }^{32}$

\section{Conclusion}

In this study, we found that DSF had significant antitumor activity in GBM cells through the induction of ferroptosis. DSF also enhanced the sensitivity of GBM cells to radiotherapy. For the first time, our study demonstrates that DSF triggers LMP in a ROS-dependent manner. ROS may thus be a key link between ferroptosis and LMP. Altogether, our study revealed DSF as a promising antitumor compound in the treatment of GBM. However, further study of these properties of DSF and other inducers of ferroptosis might also contribute to the development of novel treatments for other tumor types.

\section{Funding}

This work was supported by the Natural Science Foundation of China Grant (81702475, 81803045, and 81903126) and The Jinan Science and Technology Bureau of Shandong Province (201704083).

\section{Disclosure}

The authors report no conflicts of interest in this work.

\section{References}

1. Touat M, Idbaih A, Sanson M, Ligon KL. Glioblastoma targeted therapy: updated approaches from recent biological insights. Ann Oncol. 2017;28(7):1457-1472. doi:10.1093/annonc/mdx106

2. Hwang JS, Lee YY, Lee DH, Kwon KH. DATS sensitizes glioma cells to TRAIL-mediated apoptosis by up-regulation of death receptor 5 via ROS. Food Chem Toxicol. 2017;106(Pt A):514-521. doi:10.1016/j.fct.2017.05.056

3. Bebber CM, Muller F, Prieto Clemente L, Weber J, von Karstedt S. Ferroptosis in cancer cell biology. Cancers. 2020;12(1):164. doi: $10.3390 /$ cancers 12010164

4. Dixon SJ, Lemberg KM, Lamprecht MR, et al. Ferroptosis: an irondependent form of nonapoptotic cell death. Cell. 2012;149(5):10601072. doi:10.1016/j.cell.2012.03.042

5. Ma D, Li C, Jiang P, Jiang Y, Wang J, Zhang D. Inhibition of ferroptosis attenuates acute kidney injury in rats with severe acute pancreatitis. Dig Dis Sci. 2020. doi:10.1007/s10620-020-06225-2

6. Seibt TM, Proneth B, Conrad M. Role of GPX4 in ferroptosis and its pharmacological implication. Free Radic Biol Med. 2019;133:144152. doi:10.1016/j.freeradbiomed.2018.09.014

7. Sang M, Luo R, Bai Y, et al. BHQ-cyanine-based "off-on" longcirculating assembly as a ferroptosis amplifier for cancer treatment: a lipid-peroxidation burst device. ACS Appl Mater Interfaces. 2019;11 (46):42873-42884. doi:10.1021/acsami.9b12469

8. Yang WS, SriRamaratnam R, Welsch ME, et al. Regulation of ferroptotic cancer cell death by GPX4. Cell. 2014;156(1-2):317-331. doi:10.1016/j.cell.2013.12.010

9. Lu B, Chen XB, Ying MD, He QJ, Cao J, Yang B. The role of ferroptosis in cancer development and treatment response. Front Pharmacol. 2017;8:992. doi:10.3389/fphar.2017.00992

10. Yu M, Gai C, Li Z, et al. Targeted exosome-encapsulated erastin induced ferroptosis in triple negative breast cancer cells. Cancer Sci. 2019;110(10):3173-3182.

11. Gai C, Yu M, Li Z, et al. Acetaminophen sensitizing erastin-induced ferroptosis via modulation of Nrf2/heme oxygenase-1 signaling pathway in non-small-cell lung cancer. J Cell Physiol. 2020;235(4):33293339.

12. Lei G, Zhang Y, Koppula P, et al. The role of ferroptosis in ionizing radiation-induced cell death and tumor suppression. Cell Res. 2020;30(2):146-162. doi:10.1038/s41422-019-0263-3

13. Ye LF, Chaudhary KR, Zandkarimi F, et al. Radiation-induced lipid peroxidation triggers ferroptosis and synergizes with ferroptosis inducers. ACS Chem Biol. 2020.

14. Serrano-Puebla A, Boya P. Lysosomal membrane permeabilization as a cell death mechanism in cancer cells. Biochem Soc Trans. 2018;46 (2):207-215. doi:10.1042/BST20170130

15. Serrano-Puebla A, Boya P. Lysosomal membrane permeabilization in cell death: new evidence and implications for health and disease. Ann N Y Acad Sci. 2016;1371(1):30-44. doi:10.1111/nyas.12966

16. Gao H, Bai Y, Jia Y, et al. Ferroptosis is a lysosomal cell death process. Biochem Biophys Res Commun. 2018;503(3):1550-1556. doi:10.1016/j.bbrc.2018.07.078

17. Li Y, Chen F, Chen J, et al. Disulfiram/copper induces antitumor activity against both nasopharyngeal cancer cells and cancer-associated fibroblasts through ROS/MAPK and ferroptosis pathways. Cancers. 2020;12(1):138. doi:10.3390/cancers 12010138

18. Butcher K, Kannappan V, Kilari RS, et al. Investigation of the key chemical structures involved in the anticancer activity of disulfiram in A549 non-small cell lung cancer cell line. BMC Cancer. 2018;18 (1):753. doi:10.1186/s12885-018-4617-x

19. Park YM, Go YY, Shin SH, Cho JG, Woo JS, Song JJ. Anti-cancer effects of disulfiram in head and neck squamous cell carcinoma via autophagic cell death. PLoS One. 2018;13(9):e0203069. doi:10.1371/ journal.pone. 0203069 
20. Shah O'Brien P, Xi Y, Miller JR, et al. Disulfiram (Antabuse) activates ROS-dependent ER stress and apoptosis in oral cavity squamous cell carcinoma. J Clin Med. 2019;8(5):611. doi:10.3390/ jcm8050611

21. Zhang X, Xu R, Zhang $\mathrm{C}$, et al. Trifluoperazine, a novel autophagy inhibitor, increases radiosensitivity in glioblastoma by impairing homologous recombination. J Exp Clin Cancer Res. 2017;36 (1):118. doi:10.1186/s13046-017-0588-Z

22. Liu P, Kumar IS, Brown S, et al. Disulfiram targets cancer stem-like cells and reverses resistance and cross-resistance in acquired paclitaxel-resistant triple-negative breast cancer cells. $\mathrm{Br} J$ Cancer. 2013;109(7):1876-1885. doi:10.1038/bjc.2013.534

23. Rolle F, Bincoletto V, Gazzano E, et al. Coencapsulation of disulfiram and doxorubicin in liposomes strongly reverses multidrug resistance in breast cancer cells. Int J Pharm. 2020;580:119191. doi:10.1016/j.ijpharm.2020.119191

24. Xu X, Xu J, Zhao C, et al. Antitumor effects of disulfiram/copper complex in the poorly-differentiated nasopharyngeal carcinoma cells via activating ClC-3 chloride channel. Biomed Pharm. 2019;120:109529. doi:10.1016/j.biopha.2019.109529

25. Li H, Wang J, Wu C, Wang L, Chen ZS, Cui W. The combination of disulfiram and copper for cancer treatment. Drug Discov Today. 2020;25(6). doi:10.1016/j.drudis.2020.04.003
26. Rathore R, McCallum JE, Varghese E, Florea AM, Busselberg D. Overcoming chemotherapy drug resistance by targeting inhibitors of apoptosis proteins (IAPs). Apoptosis. 2017;22(7):898-919.

27. Conrad M, Kagan VE, Bayir H, et al. Regulation of lipid peroxidation and ferroptosis in diverse species. Genes Dev. 2018;32(910):602-619.

28. Zhang X, Hu P, Ding SY, et al. Induction of autophagy-dependent apoptosis in cancer cells through activation of ER stress: an uncovered anti-cancer mechanism by anti-alcoholism drug disulfiram. Am J Cancer Res. 2019;9(6):1266-1281.

29. Cai X, Liu Y, Hu Y, et al. ROS-mediated lysosomal membrane permeabilization is involved in bupivacaine-induced death of rabbit intervertebral disc cells. Redox Biol. 2018;18:65-76.

30. Wang F, Gomez-Sintes R, Boya P. Lysosomal membrane permeabilization and cell death. Traffic. 2018;19(12):918-931. doi:10.1111/ tra. 12613

31. Zhang X, Ding K, Ji J, et al. Trifluoperazine prolongs the survival of experimental brain metastases by STAT3-dependent lysosomal membrane permeabilization. Am J Cancer Res. 2020;10(2):545-563.

32. Kirtonia A, Gala K, Fernandes SG, et al. Repurposing of drugs: an attractive pharmacological strategy for cancer therapeutics. Semin Cancer Biol. 2020. doi:10.1016/j.semcancer.2020.04.006

\section{Publish your work in this journal}

OncoTargets and Therapy is an international, peer-reviewed, open access journal focusing on the pathological basis of all cancers, potential targets for therapy and treatment protocols employed to improve the management of cancer patients. The journal also focuses on the impact of management programs and new therapeutic agents and protocols on patient perspectives such as quality of life, adherence and satisfaction. The manuscript management system is completely online and includes a very quick and fair peer-review system, which is all easy to use. Visit http://www.dovepress.com/ testimonials.php to read real quotes from published authors. 